\title{
LA POLÍTICA DE ENRIQUE PEÑA NIETO HACIA AMÉRICA DEL NORTE: NORMALIZACIÓN E INSTITUCIONALIZACIÓN
}

\author{
THE POLITICS OF ENRIQUE PEÑA NIETO AND \\ NORTH AMERICA: NORMALIZATION \\ AND INSTITUTIONALIZATION
}

\section{LA POLITIQUE D'ENRIQUE PEÑA NIETO VIS-À-VIS DE L'AMERIQUE DU NORD: NORMALISATION ET INSTITUTIONNALISATION}

Arturo Santa Cruz

Universidad de Guadalajara* arturosc@hotmail.com

Resumen: Este trabajo tiene como objetivo presentar una panorámica general de la política exterior del gobierno de Enrique Peña Nieto hacia América del Norte, de importancia central debido al proceso de normalización de la cooperación en materia de seguridad con Washington. Asimismo, la defensa de la relativamente reciente institucionalización de la relación económica con Estados Unidos y Canadá es también relevante. Para abordar estos temas, se revisan brevemente algunos conceptos analíticos que servirán para anclar la narrativa; en la segunda sección se examina la relación de México con Estados Unidos con foco en las dos áreas temáticas antes mencionadas. En la tercera sección se hace un breve repaso de la relación con Canadá; en la cuarta se trata sucintamente la política de México hacia América del Norte en su conjunto y, a manera de conclusión, se presenta un breve señalamiento sobre el futuro de la región.

Palabras clave: América del Norte; Canadá; Estados Unidos; política exterior; México; seguridad; relación económica.

Aвstract: This article aims to present a general overview of the foreign policy of the government of Enrique Peña Nieto with regard to North America,

* Expreso mi agradecimiento a Christian Cabrera por su muy valiosa asistencia de investigación en la elaboración de este trabajo. 
which is of importance due to the process of normalization of cooperation on security with Washington. Likewise, the defense of the relatively recent institutionalization of the economic relationship with the United States and Canada is also significant. To address these issues, a number of analytical concepts that will serve to anchor the narrative are briefly reviewed; the second section examines Mexico's relationship with the United States, focusing on the two aforementioned thematic areas. The third section briefly reviews the relationship with Canada; the fourth deals succinctly with Mexico's policy towards North America as a whole and, as a conclusion, presents a brief statement on the future of the region.

Keywords: North America; Canada; United States; foreign policy;

Mexico; security; economic relationship.

\section{Traducción de Gonzalo Celorio Morayta}

Résumé: Cet article trace un panorama de la politique d'Enrique Peña Nieto vis-à-vis de l'Amérique du Nord, dans le cadre d'une normalisation de la coopération avec Washington en matière de sécurité. La défense de l'institutionnalisation assez récente des rapports économiques avec les États-Unis et le Canada est également importante. Pour mieux aborder ces sujets, on passe d'abord en revue quelques concepts qui serviront de base à l'analyse. La seconde partie de l'article est consacrée aux rapports Mexique-États-Unis, surtout pour ce qui est de la sécurité et de l'économie. La troisième évoque les relations avec le Canada, tandis que la dernière propose un bilan de la politique du Mexique envers ses voisins du Nord. Dans les conclusions on s'interroge sur l'avenir possible de la région.

Mots clefs: Amérique du Nord; Canada; États-Unis; politique étrangère;

Mexique; sécurité; rapports économiques.

Traducción de BERNARDo MABIRE

Fecha de recepción: diciembre de 2018

Fecha de aceptación: febrero de 2019 


\section{E} L gobierno de Enrique Peña Nieto inició la relación con Estados Unidos -la más importante para México en América del Norte-, tratando de reencuadrarla, de "cambiar la narrativa". Después de la grave crisis de seguridad vivida por nuestro país durante el sexenio de Felipe Calderón (con una tasa de homicidio de 22/100 000 en el último año de su gobierno, contra poco menos de 10/100000 seis años antes), ${ }^{1}$ la cual en buena medida definió la relación bilateral debido a la cooperación iniciada en el marco de la Iniciativa Mérida, Peña Nieto intentó cambiar el foco del trato con el vecino del norte, y puso énfasis en el aspecto económico. ${ }^{2}$ La intención del nuevo gobierno parecía ir en serio. De entrada, el secretario de Gobernación peñanietista, Miguel Ángel Osorio Chong, estableció la "ventanilla única" en su ministerio, a fin de centralizar los contactos en materia de seguridad con los estadounidenses. Más adelante declararía que el programa emblema de la cooperación binacional en la materia, la Iniciativa Mérida, estaba "en su fase terminal". ${ }^{3}$

Por otra parte, en congruencia con su plan de reencuadrar la relación con Washington, cinco meses después de asumir el poder, en conferencia de prensa conjunta en la Ciudad de México con el presidente estadounidense Barack Obama, el presidente Peña anunció la creación del Diálogo Económico de Alto Nivel (DEAN). Durante ésta, tal como lo consignó The New York Times, los mandatarios "repetidamente intentaron cambiar su conversación pública, alejándose de los temas fronterizos y de seguridad [...] para enfocarse en cambio en la vasta relación económica entre ambas naciones". ${ }^{4}$

${ }^{1}$ A. Hope, "Seis apuntes sobre un sexenio fallido", El Universal, 26 de noviembre de 2018; Institute for Economics and Peace, "Índice de datos sobre homicidios", 2018, p. 6.

${ }^{2}$ R. Archibold y E. Malkin, "Mexico Shifts Focus from Drug War to Economy", The New York Times, 27 de noviembre de 2012.

${ }^{3}$ L. López, "Iniciativa Mérida entregó 2.3 mil mdd en siete años", Grupo Milenio, 2 de febrero de 2017.

${ }^{4}$ M. Shear y R. Archibold, "Obama Arrives in Mexican Capital to Meet With New Leader”, The New York Times, 2 de mayo de 2013. 
De manera aparente, pues, la estrategia del nuevo presidente mexicano estaba funcionando, y digo de manera aparente porque la realidad es terca y difícil de moldear con el mero discurso. Para empezar, aparte del cambio de énfasis en materia de seguridad, la crisis seguía allí, y su superación, que constituyó uno de los temas centrales de la campaña presidencial de Peña Nieto, parecía requerir todavía del apoyo estadounidense. Incluso en varias instancias del gobierno mexicano existía interés en que ésta continuara. Así, el Rubicón que en materia de seguridad se había cruzado el sexenio anterior con la Iniciativa Mérida, parecía ser una frontera ya rebasada. ${ }^{5}$ En la demarcación del nuevo territorio de cooperación binacional, es decir, en la normalización de la frontera de política pública trascendida durante el gobierno anterior, ciertas redes intergubernamentales tendrían un papel significativo.

Por otra parte, si bien con el DEAN se consiguieron algunos avances importantes en materia económica (mayor información más adelante), ciertamente no funcionó como un punto focal de la relación bilateral. En el ámbito comercial, ese papel lo tendría la negociación del Acuerdo Transpacífico, el cual era, como varios actores y analistas señalarían, una renegociación indirecta, o de facto, del Tratado de Libre Comercio de América del Norte (TLCAN), con Barack Obama en la Casa Blanca. Más adelante, la renegociación del TLCAN se realizaría, ahora sí directamente, ya con Donald Trump instalado en la oficina oval. Curiosamente, durante el segundo momento recién mencionado, el gobierno del presidente Peña Nieto se ocupó también de una frontera de política pública importante para la relación bilateral, que es la formalización de la integración económica con Estados Unidos; en esta ocasión, sin embargo, ante el ánimo proteccionista del gobierno de Trump, el gobierno mexicano tenía como

${ }^{5}$ A. Santa Cruz, "La política exterior de Felipe Calderón hacia América del Norte: crisis interna y redefinición de fronteras". Foro Internacional, 53 (2013), pp. 537-571. 
objetivo la defensa de la frontera establecida en el ámbito económico dos décadas atrás.

En este contexto, el objetivo del presente trabajo es presentar una panorámica general de la política exterior del gobierno de Enrique Peña Nieto hacia América del Norte. Debido a la importancia del proceso de normalización de la cooperación en materia de seguridad con Washington, máxime cuando la intención expresa del gobierno peñanietista era revertirla, este tema ocupa un lugar importante en este trabajo, lo mismo que la defensa de la relativamente reciente institucionalización de la relación económica con Estados Unidos -y Canadá-, de modo que se revisan algunos conceptos analíticos como la frontera de política pública, las redes transgubernamentales y el encuadre. En la segunda sección la revisión de la relación de México con Estados Unidos se centra en la seguridad y la economía, dejando fuera (por motivos de espacio) temas importantes de la relación bilateral, como la migración o el muro fronterizo. En la tercera se hace un breve repaso de la relación con Canadá para en la cuarta tratar sucintamente la política de México hacia América del Norte en su conjunto y concluir con un señalamiento breve del futuro de la región.

Fronteras de políticas públicas, RELACIONES TRANSGUBERNAMENTALES Y ENCUADRE

En lo referente al concepto "fronteras de políticas públicas" cito a Stephanie Golob, quien las define como "barreras erigidas por concepciones sagradas, mantenidas históricamente, de la soberanía, la seguridad y la identidad nacional, que hacen que ciertas alternativas no sean consideradas como opciones entre el repertorio de políticas públicas «normales»" ${ }^{6}$ El caso de la cooperación en seguridad, trata sobre una "fron-

${ }^{6}$ S. Golob, "Beyond the Policy Frontier: Canada, Mexico, and the Ideological Origins of NAFTA", World Politics 55 (2003), p. 361. 
tera" en construcción, como lo evidencia la inicial reacia actitud del gobierno peñanietista a seguir cooperando con Washington en la materia. ${ }^{7} \mathrm{El}$ "instinto" del nuevo gobierno procedente del Partido Revolucionario Institucional (PRI) era regresar a lo que por décadas había sido una política pública "normal". Sin embargo, lo interesante del caso es que, hasta cierto punto, la colaboración con Washington ya se había "normalizado", si bien no arraigándose en el imaginario nacional como lo había hecho la (formalmente) distante relación económica con Estados Unidos en el periodo revolucionario o, en sentido contrario, institucionalizando la relación en el mismo ámbito más recientemente. Las políticas públicas, como la identidad son, después de todo, maleables.

La incipiente normalización en la colaboración en materia de seguridad con el vecino del norte operó, como lo sugerí anteriormente, por medio de redes transgubernamentales, esto es, mediante el contacto frecuente y cercano entre ciertos funcionarios de ambos lados de la frontera. Geoffrey Hale define las relaciones transgubernamentales como la "extensa interacción transfronteriza entre funcionarios ejecutivos, legislativos, administrativos y reguladores de gobiernos nacionales y, a veces, subnacionales, fuera de los procesos diplomáticos formales centrados en los ministerios de relaciones exteriores". ${ }^{8}$ En el caso en cuestión, como se verá más adelante, la red transgubernamental relevante es la encargada del aparato de seguridad estatal, en sentido amplio. El concepto de relaciones transnacionales está, por supuesto, íntimamente relacionado con la teoría de la interdependencia en la disciplina de relaciones internacionales y, como señalaban dos de sus autores pioneros, Robert Keohane y Joseph Nye, una vez que la interdependencia se establece entre dos o

7 T. Wilkinson, "Mexico captures drug kingpin's father-in-law", Los Angeles Times, 30 de abril de 2013.

${ }^{8}$ G. Hale, "Transnationalism, Transgovernmentalism and Canada-US Relations in the Twenty-first Century", American Review of Canadian Studies, 43 (2013), p. 497. 
más Estados en ciertos temas, no es raro que se propague a otras. ${ }^{9}$ Sin afirmar que haya una relación causal, el antecedente de la formalización de la interdependencia económica entre México y Estados Unidos, dos décadas antes de la formalización de la cooperación bilateral en materia de seguridad es, al menos, sugerente. Todavía más, la defensa de la frontera de política pública en comento apunta hacia la necesidad, al menos en ocasiones, de remplazar el enfoque disciplinario que considera al Estado como un actor unitario por otro que lo desagregue, pues es evidente que en ocasiones ciertos nichos burocráticos pueden impulsar, o incluso lograr por su cuenta, coordinación interestatal en su área temática. $^{10}$

Lo anterior me lleva al último término analítico que quiero introducir: el encuadre. De acuerdo con Alex Mintz y Steven Redd, el encuadre intencional "es un intento por parte de líderes u otros actores influyentes para introducir en el debate de políticas públicas (o en la deliberación grupal) temas organizativos que afectarán cómo los objetivos mismos, así como el público y otros actores (por ejemplo, los medios de comunicación) perciben un problema". ${ }^{11}$ Así, como señala David Snow, "al hacer que los eventos o sucesos adquieran significado, los encuadramientos sirven para organizar la experiencia y guiar la acción, sea ésta individual o colectiva”. ${ }^{12}$ La relación del concepto de encuadre con la discusión anterior es que, a pesar del intento del gobierno del presidente

${ }^{9}$ R. Keohane y J. S. Nye. (eds.), Transnational Relations and World Politics, Cambridge, Harvard University Press, 1972; R. Keohane y J. Nye, Power and Interdependence: World Politics in Transition, Boston, Little, Brown and Co., 1977.

${ }^{10}$ A. Slaughter, "The Real New World Order”. Foreign Affairs, 76, núm. 5 (1997), pp. 183-197.

11 A. Mintz, y S. Redd, "Framing Effects in International Relations", Synthese 135, núm 2 (2003), p. 194.

12 M. Keck, y Sikkink K, "Transnational Advocacy Networks in the Movement Society", en D. Meyer y S. Tarrow (eds.), The Social Movement Society: Contentious Politics for a New Century, Lanham, Rowman \& Littlefield, 1998, pp. 217-238. 
Peña Nieto por reencuadrar el problema tanto de la cooperación en materia de seguridad con Estados Unidos, como del problema mismo en nuestro país (matizando la dimensión de este último y presentando un "nuevo" enfoque -la famosa "coordinación" entre los diferentes niveles de gobierno y agencias gubernamentales involucrados en la problemática), los límites de la nueva narrativa se mostraron pronto. No es solamente que ni la cooperación con el vecino del norte haya disminuido (más bien al contrario) ni que el nivel de violencia en el país haya hecho lo propio (al contrario, el sexenio peñanietista cerró con una tasa de homicidio de 27 por 100000 habitantes $)^{13}$ sino que, ante la terca realidad, la nueva narrativa careció de credibilidad y, por lo tanto, de eficacia. ${ }^{14}$

Normalizando la FRONTERA DE POLÍtica PÚblica DE COOPERACIÓN EN SEGURIDAD CON WASHINGTON

El regreso del PRI a Los Pinos, después de 12 años de gobiernos emanados del Partido Acción Nacional, planteaba la incógnita sobre el futuro, no sólo de la Iniciativa Mérida, sino de la cooperación en materia de seguridad en la relación con Estados Unidos. Como ha observado Craig Deare, exconsejero de Seguridad Nacional para el Hemisferio Occidental de Donald Trump, "la gran interrogante era si los nuevos niveles de cooperación desarrollados durante el sexenio de Calderón continuarían”. ${ }^{15}$ Veníamos, habría que recordar, de lo que Arturo Valenzuela, subsecretario de Estado para Asuntos Hemisféricos al inicio del gobierno de Barack Obama, había

${ }^{13}$ A. Hope, "Seis apuntes sobre un sexenio fallido", El Universal, 26 de noviembre de 2018.

14 Véase V. Romero, B. Magaloni, A. Díaz-Cayeros, "The Mexican War on Drugs: Crime and the Limits of Government Persuasion", International Journal of Public Opinion Research, núm. 27 (2015), p. 127.

15 C. Deare, A Tale of Two Eagles: The US-Mexico Bilateral Defense Relationship Post Cold War, Lanham, Rowman \& Littfield, 2017, p. 354. 
llamado un "hito" en el cambio operado en materia de seguridad, de alguna manera equiparable, según él, a la firma del TLCAN dos décadas atrás. ${ }^{16}$

Como sugerí anteriormente, con la "ventanilla única", la intención del gobierno peñanietista era centralizar las decisiones de cooperación con Washington. En palabras del secretario Osorio Chong: "No se vale, ni se podrá, ni se permitirá que cada agencia determine con quién se entiende. Así se venía realizando antes [...]. Ahora hay un solo conducto, es Gobernación”. ${ }^{17}$ Sin embargo, como ha notado Íñigo Guevara: "El cambio sólo impactó las agencias civiles de seguridad, puesto que la recientemente institucionalizada relación bilateral entre las fuerzas armadas mexicanas y estadounidenses permaneció relativamente inalterada". ${ }^{18}$ Todavía más: la cooperación perduró no sólo más allá del ámbito civil, sino también más allá del ámbito geográfico del gobierno federal, pues en las zonas fronterizas la cooperación entre los agentes de los dos países persistió. ${ }^{19}$

Ya desde el gobierno anterior había operado una red transgubernamental en materia de seguridad que, haciendo el símil con las redes de defensa transnacional en la literatura sobre la emergencia y difusión de las normas, se puede afirmar que recurrió al efecto boomerang, como estas últimas lo hacen. ${ }^{20}$ En el caso de la red transgubernamental en cuestión, los servicios de inteligencia mexicanos, concretamente

${ }^{16}$ Arturo Valenzuela, entrevista con el autor, Guadalajara, Jalisco, $1^{\circ}$ de diciembre de 2012.

17 "México anuncia nuevo modelo «centralizado" de seguridad con EEUU", La Opinión, 2 de mayo de 2013, http://www.laopinion.com/2013/ 05/02/mexico-anuncia-nuevo-modelo-centralizado-de-seguridad-coneeuu/ (consulta del 5 de noviembre de 2018).

${ }^{18}$ I. Guevara Moyano, "A Bond Worth Strengthening: Understanding the Mexican Military and U.S.-Mexican Military Cooperation", Wilson Center Mexico Institute, 11 de octubre de 2016.

${ }^{19}$ A. Selee, Vanishing Frontiers: The Forces Driving Mexico and the United States Together. Nueva York, Public Affairs, 2018, pp. 156, 178.

${ }^{20}$ M. Keck y K. Sikkink, Activists Beyond Borders: Advocacy Networks in International Politics, Ithaca, Cornell University Press, 1998. 
el Cisen, recurrió al estadounidense Comando del Norte (NORTHCOM), para que éste planteara a SEDENA el establecimiento de los Centros de Fusión, a los cuales ésta se oponía y CISEN favorecía; al final, los centros fueron establecidos. ${ }^{21}$ Así, los militares estadounidenses se convirtieron en interlocutores privilegiados de los servicios de inteligencia y seguridad mexicanos, particularmente de estos últimos. Tanto el general Chuck Jacoby, comandante del NORTHсом, como Eduardo Medina Mora, exembajador de México en Washington, han notado que las fuerzas castrenses mexicanas preferían tener contacto con la parte militar -que con la civil- de la Secretaría de Defensa de Estados Unidos. ${ }^{22}$ Así pues, independientemente de las directivas establecidas por el gobierno peñanietista para esos años, como Alejandro Hope ha señalado, la norma de la cooperación binacional estaba ya "internalizada" por las instancias mexicanas de seguridad. ${ }^{23}$

De esta manera, aunque por el discurso del gobierno de Enrique Peña Nieto se temía que la Iniciativa Mérida llegara a su fin, ésta continuó y evolucionó. Así, por ejemplo, México recibió más de 850 millones de dólares de Estados Unidos en el marco de este programa entre 2013 y $2018 .{ }^{24}$ Respecto a la evolución del esquema de cooperación, al amparo de éste se mejoró el sistema penitenciario en nuestro país, pues se acreditaron 17 centros de detención. ${ }^{25}$ Más relevante es el hecho de que los servicios de inteligencia estadounidenses hayan estado involucrados en algunas de las más importan-

${ }^{21}$ C. Deare, op. cit., p. 300.

22 Ibid., pp. 326, 339.

${ }^{23}$ A. Hope, Plus Ca Change: Structural Continuities in Mexican Counternarcotics Policy, Washington, DC, Brookings Institution, Latin American Initiative, 2016.

${ }^{24}$ C. Ribando Seelke y K. Finklea. "U.S. Mexican Security Cooperation: The Merida Initiative and Beyond", Congressional Research Service, junio 29, 2017, p. 11. Las cifras en millones de dólares por año fueron: 222.2 en 2013, 178.1 en 2014, 143.6 en 2014, 139 en 2016, 139 en 2017 y 85 en 2018.

${ }^{25}$ D. Baptista, "Amarran fondos de Iniciativa Mérida", Mural, 29 de enero de 2017. 
tes detenciones de líderes del crimen organizado que tuvieron lugar durante la administración peñanietista, como fueron los casos de Inés Coronel, suegro de Joaquín el Chapo Guzmán, de Miguel Ángel Treviño Morales, el Z-40, y del mismo Chapo (en dos ocasiones: febrero de 2014 y enero de 2016); lo anterior es prueba fehaciente de que la cooperación en la materia continuó durante el gobierno de Peña Nieto. ${ }^{26}$ Como notara The Economist, apenas 15 meses después del inicio de dicho mandato, -en el contexto de la primera captura del Chapo- "existe más colaboración entre México y Estados Unidos de la que es aparente". ${ }^{27}$

Más allá de la Iniciativa Mérida fueron ilustrativos también los frecuentes contactos entre los sectores de seguridad de México y Estados Unidos. Así, por ejemplo, se estableció el Grupo Bilateral de Cooperación en Seguridad, el cual llevó a cabo cinco reuniones. ${ }^{28}$ Así, no es de extrañar que a inicios de febrero de 2016, Jeh Johnson, secretario de Seguridad Interior del gobierno de Obama, se congratulara por el buen estado de la cooperación bilateral en la materia, señalando que se había reunido más de diez veces con miembros del gabinete de seguridad de Peña Nieto. ${ }^{29}$ Los sectores castrenses de México y Estados Unidos también mantuvie-

${ }^{26}$ R. C. Archibold y G. Thompson, "Capture of Mexican Crime Boss Appears to End a Brutal Chapter", The New York Times, 16 de julio de 2013; "EU, tras la caída de El Z-40", La Jornada, 18 de julio de 2013; Jorge Carrillo Olea, "Tres-espionajes-tres", La Jornada, 6 de septiembre de 2013; J. Partlow y S. Horwitz, "U.S. and Mexican authorities detail coordinated effort to capture drug lord", Washington Post, 23 de febrero de 2014; R. Riva Palacio, "La captura de la abuela”, El Financiero, 3 de marzo de 2017.

${ }^{27}$ H.T. "Got Shorty", The Economist, 22 de febrero de 2014.

28 Secretaría de Relaciones Exteriores, "América del Norte: una agenda trilateral para construir prosperidad compartida", Embajada de México en Estados Unidos, Comunicado núm. 656, 27 de diciembre de 2015; Secretaría de Relaciones Exteriores, "Se lleva a cabo la V Reunión del Grupo Bilateral de Cooperación en Seguridad México-Estados Unidos, Comunicado de prensa, 17 de octubre de 2017.

${ }^{29}$ E. Hernández, "Destaca Presidente Integración con EEuU”, Mural, 5 de febrero de 2016 , p. 5. 
ron un estrecho contacto durante el gobierno de Peña Nieto; el caso es que en abril de 2017 se celebró por primera vez, en suelo mexicano, la Conferencia de Seguridad de Centroamérica, a la que asistieron los altos mandos militares estadounidenses. ${ }^{30}$

Otro hecho por demás significativo del cambio operado en la relación bilateral en materia de seguridad durante el sexenio que nos ocupa es que en septiembre de 2017, en pleno gobierno de Donald Trump -presidente durante cuyo mandato la relación bilateral ha estado en permanente estrés debido a los constantes ataques del mandatario a nuestro país-, la Secretaría de Defensa haya invitado a Jim Mattis, secretario de Defensa de Estados Unidos, a participar en la celebración de la Independencia. Además, como hizo notar el propio General retirado, el único desfile con honores que él había autorizado desde que asumió el cargo fue para los dos socios de su país en el TLCAN. ${ }^{31}$ En el mismo sentido, tanto el secretario de Marina, Vidal Francisco Soberón, en 2015, como el secretario de Defensa, Salvador Cienfuegos, en 2018, recibieron en la National Defense University, en Washington, el premio William J. Perry (el de Defensa durante el gobierno de Bill Clinton). ${ }^{32}$ Aun en el enrarecido ambiente del gobierno de Trump, la relación bilateral entre las fuerzas armadas de los dos países es descrita por funcionarios estadounidenses de alto rango como "un oasis". ${ }^{33}$ En efecto, de acuerdo con el ya citado exconsejero de Seguridad

30 D. Estévez, "Cumbre militar en Cozumel, escenario del debut de México como líder regional”, Sin Embargo, 21 abril de 2017, http:/ / www. sinembargo.mx/author/dolia_estevez

${ }^{31}$ J. Faus, "El jefe del Pentágono viaja a México para celebrar el Día de la Independencia", El País, 15 de septiembre de 2017.

32 "Reconocen en Eu a Secretario de Marina", Reforma, 15 de diciembre de 2015; "Frentes Políticos", Excélsior, 21 de septiembre de 2018; "Salvador Cienfuegos recibe premio por contribuir a garantizar la seguridad", El Universal, 20 de septiembre de 2018.

${ }^{33}$ I. Guevara, "Análisis: La relación militar entre México-Eu, un oasis en la era Trump”, El Heraldo de México, $1^{\circ}$ mayo de 2017. 
Nacional para el Hemisferio Occidental del actual presidente estadounidense, la relación de defensa entre los dos países ha mejorado respecto al sexenio de Calderón. ${ }^{34}$

Dos casos más ilustran el nivel al que ha llegado la cooperación entre México y Estados Unidos. Uno es el revelado en abril de 2018 por The Washington Post en materia migratoria. De acuerdo con lo publicado por el rotativo, personal estadounidense de la Secretaría de Seguridad Interior tiene acceso en México a los centros de detención para migrantes, en donde obtienen datos biométricos de los detenidos (se han obtenido datos de alrededor de 30000 migrantes). De acuerdo con la nota, si bien este programa se acordó en 2012 durante el gobierno calderonista, no se echó a andar sino hasta dos años después. ${ }^{35} \mathrm{El}$ segundo caso se refiere a la creación, en agosto de 2018, de un equipo de investigación conjunto de la Procuraduría General de la República y la Drug Enforcement Administration (DEA) para combatir las redes financieras del crimen organizado, con sede en Chicago. De manera significativa, durante el anuncio hecho en la ciudad estadounidense estuvieron presentes representantes de SEDENA. ${ }^{36}$ Así pues, está claro que la relación de seguridad ha trascendido a la Iniciativa Mérida, al crearse una red transgubernamental de seguridad que se ha institucionalizado y extendido a una agenda más diversa.

\section{DEFENDIENDO LA FRONTERA DE POLÍTICA PÚBLICA DE LIBRE COMERCIO GON ESTADOS UNIDOS}

Como señalé anteriormente, desde el inicio de su gobierno el presidente Peña Nieto intentó reencuadrar la relación del

${ }^{34}$ Entrevista con el autor, Guadalajara, 19 de octubre de 2018.

35 J. Partlow y N. Miroff, "U.S. gathers data on migrants deep in Mexico, a sensitive program Trump's rhetoric could put at risk", The Washington Post, 6 de abril de 2018.

${ }^{36}$ R. Mosso, "PGR y DEA, contra las redes financieras del narco en EU", Milenio, 16 de agosto 2018, p. 6. 
país con Estados Unidos y, para este fin, se creó (el 2 de mayo de 2013), el ya mencionado Diálogo Económico de Alto Nivel (DEAN). Más allá del intento de dirigir la atención de la intensa relación bilateral al ámbito económico, el DEAN fue un intento de preservar la frontera que representaba la integración económica regional establecida en los años noventa. Entre las áreas prioritarias de trabajo del grupo binacional estaban la cooperación regulatoria, el desarrollo de la fuerza laboral, la energía e infraestructura fronteriza. El DEAN, como apuntó Penny Pritzker, secretaria de Comercio del presidente Obama, "representa el siguiente paso hacia adelante en la relación económica Estados Unidos-México". ${ }^{37}$

En buena medida gracias al alto nivel de liderazgo que lo guiaba, incluso sin ser un proyecto de gran calado, el DEAN sirvió para obtener logros importantes. Entre los resultados que vale la pena destacar se encuentran la facilitación del tránsito de mercancías mediante el reconocimiento mutuo de empresas certificadas, la implementación de programas piloto de preinspección de carga y el incremento sustancial de rutas aéreas y de fletes entre los dos países (gracias al logro de un acuerdo de servicios aéreos), así como del padrón de viajeros confiables. Asimismo, el establecimiento del Foro Bilateral sobre Educación Superior, Innovación e Investigación (FOBEsII), en el marco del cual, por ejemplo, se signaron más de un centenar de acuerdos entre instituciones de educación de México y Estados Unidos. ${ }^{38}$ De manera similar, mediante los programas Proyecta 100,000 e iniciativa La Fuerza de los 100,000 en las Américas, se incrementó sustancialmente el número de estudiantes mexicanos en el vecino país y de alumnos estadounidenses en el nuestro.

En lo referente a la cooperación en materia energética e infraestructura fronteriza, cabe destacar tanto el acuerdo para

37 P. Pritzker, “Opinion Editorial: US-Mexico Economic Talks are Key to Cross-Border Prosperity", 6 de enero de 2015, U.S. Department of Commerce (sitio de internet), https://www.commerce.gov

38 "Continúa Diálogo Económico de Alto Nivel entre México y EU”, El Economista, 9 de diciembre de 2016. 
el uso pacífico de la energía nuclear (que tiene el potencial de incrementar las exportaciones mexicanas de bienes de ese sector como de mejorar ambientalmente el desempeño del sector energético), como la construcción del Cruce Ferroviario Matamoros-Brownsville, el cruce peatonal Puerta Este, en Tijuana-San Ysidro, y el puente peatonal que conecta al aeropuerto de Tijuana con San Diego.

Pero más allá del abordaje de temas económicos bilaterales específicos en el marco del DEAn, el gobierno de Peña Nieto dio seguimiento a un proyecto, éste sí de largo alcance, iniciado en las postrimerías del gobierno de Felipe Calderón: la negociación del Acuerdo Estratégico Transpacífico de Asociación Económica, conocido como Acuerdo Transpacífico o simplemente TPP, por sus siglas en inglés. México se incorporó a las pláticas cuya conclusión sería un acuerdo entre 12 países en junio de 2012; al estar en la mesa sus dos socios norteamericanos, las negociaciones transpacíficas sirvieron, en la práctica, para actualizar el TLCAN. Temas como comercio digital y prácticas regulatorias, que habían quedado fuera del acuerdo norteamericano, serían incluidos en el nuevo, más comprehensivo, acuerdo transpacífico. Como afirmó el analista canadiense Peter Clark por aquellas fechas: “¿Es el TTP una re-negociación del NAFTA [TLCAN]? Técnicamente no, prácticamente, sí." ${ }^{9}$ Las negociaciones del TTP concluyeron exitosamente en febrero de 2016. Sin embargo, antes de que éste pudiera ser ratificado por todas las partes, en Estados Unidos llegó al poder Donald Trump, quien durante su campaña electoral lo había atacado fuertemente; por lo tanto, una de sus primeras acciones como presidente fue firmar un memorando retirando a su país del Acuerdo Transpacífico.

Antes de tomar posesión Trump también había criticado enérgicamente el TLCAN -“el peor tratado jamás firma-

${ }^{39}$ P. Clark, "Is the Trans Pacific Partnership a re-writing of NAFTA? Is the Trans Pacific Partnership a re-writing of NAFTA?", iPolitics, 10 de enero de 2012. 
do" por su país- ${ }^{40}$ y prometió renegociarlo o abandonarlo. El mandatario cumplió la versión más moderada de su promesa (la renegociación) y, a ocho meses de su llegada a la Casa Blanca, iniciaron las pláticas entre Canadá, México y Estados Unidos. El proceso renegociador fue por demás tortuoso; estuvo plagado tanto de amenazas por parte de Washington de abandonar las negociaciones como de demandas absurdas. El proceso llegó a su fin el 30 de septiembre de 2018, cuando los representantes de los tres países anunciaron la conclusión exitosa de las negociaciones (previamente, el 27 de agosto, México y Estados Unidos habían llegado a un acuerdo bilateral). El acuerdo, llamado en México T-MEC, fue firmado por los tres mandatarios en Buenos Aires, en el marco de la Cumbre del Foro de Cooperación de Asia-Pacífico (APEC), el 30 de noviembre -la víspera de que feneciera el gobierno de Enrique Peña Nieto.

Es de destacar que, en las últimas etapas de la negociación, la defensa de la frontera de política pública integracionista establecida dos décadas atrás estuvo a cargo no sólo del gobierno peñanietista, sino también del gobierno entrante, encabezado por el triunfador de las elecciones celebradas el 1 de julio, Andrés Manuel López Obrador (AMLO). Afirmo que el hecho es de destacar porque tanto AMLO como buena parte de su equipo y de sus seguidores habían sido férreos opositores al TLCAN. Jesús Seade, quien se integró al equipo negociador mexicano representando al gobierno entrante, tuvo un papel importante y quedó satisfecho con el acuerdo final; para él, el "T-MEC es $20 \%$ mejor que el NAFTA". ${ }^{41}$ El propio presidente electo calificó al

${ }^{40}$ Lee Berthame, "Trudeau, Mexican president stand up for free trade, take shots at Trump-style protectionism", The National Post, 28 de junio de 2016.

${ }^{41} \mathrm{~J}$. Tolentino, "Seade: T-MEc no fue el mejor tratado, pero evitó una crisis financiera”, Expansión, 23 de octubre de 2018; C. Ochoa y E. Gut, "T-MEc es 20\% mejor que el Tlcan: Seade", Milenio, 22 de octubre de 2018. 
nuevo acuerdo como "benéfico" para los tres países de América del Norte. ${ }^{42}$

Es de destacar también que el equipo negociador mexicano, liderado por el secretario de Economía, Idelfonso Guajardo, no sólo mostró un alto grado de profesionalismo, sino también de oficio político, al construir alianzas con amplios sectores económicos y sociales de Estados Unidos que se oponían a la intransigente posición de su gobierno. ${ }^{43} \mathrm{Al}$ final del día, a pesar de que hubo algunos cambios importantes, sobre todo en lo referente a la industria automotriz (que al menos $75 \%$, en contraste con $62.5 \%$ previo, del automotor sea producido en la región para estar libre de arancel, o que el menos $40 \%$ del vehículo sea producido por trabajadores que ganan al menos 16 dólares por hora), el tratado revisado no es tan diferente del original. ${ }^{44}$ Como era de preverse -claro, suponiendo que el desquiciado mandatario estadounidense no llevara a cabo su promesa de abandonar el acuerdo, como estuvo a punto de hacer cuando cumplió 100 días en el poder- ${ }^{45}$ buena parte del T-MEC refleja lo que ya se había negociado en el marco del TTP. ${ }^{46}$ Así lo confirmó Ke-

42 E. Cervantes, "Tuvo Trump actitud abierta y tolerante-AmLO", Refor$m a, 1^{\circ}$ de octubre de 2018.

43 B. Allen, "Trump's Trade Proposals Are so Obviously Unpopular They Give Mexicans Hope”, Foreign Policy, 26 de octubre de 2017.

${ }^{44}$ D. Lynch y H. Long, "Analysts say Trump may be overly optimistic on new North American trade deal", The Washington Post, $1^{\circ}$ de octubre de 2018; J. Tankersley, "Trump Just Ripped Up NAFTA. Here's What's in the New Deal", The New York Times, 1 de octubre de 2018; G. Ritchie, "NAFTA gets a new name, but little else has changed", The Globe and Mail, $1^{\circ} \mathrm{de}$ octubre de 2018.

${ }^{45}$ J. Ivison, "White House advisors called Ottawa to urge Trudeau to help talk Trump down from scrapping NAFTA", National Post, 8 de mayo de 2018; "Medió Kushner entre Canadá y Eu por TLC", Mural, 9 de mayo de 2017.

${ }^{46}$ G. Vega y F. Campos, "La integración económica en América del Norte ante la elección de Donald Trump: un análisis prospectivo sobre el futuro del TLCAN", Foro Internacional, vol. 57, núm. 230 (2017), pp. 781-836; J. Schlesinger, W. Mauldin, "NAFTa Partners Prolong Talks Into Next Year”, The Wall Street Journal, 17 de octubre de 2017; K. Rapoza, "NAFTA Will Live Another Day, And Be Based On Obama's ттP”, Forbes, 14 de abril de 2017. 
nneth Smith, jefe de la negociación técnica por parte de México, "en capítulos de modernización, como pyMEs, buenas prácticas regulatorias, los temas de comercio digital... trabajamos con base en lo que habíamos hecho en el TTP”. 47

El nuevo TLCAN debe ratificarse ahora por los poderes legislativos de los tres países, pero donde su destino es más incierto es en Estados Unidos, pues el control del Partido Demócrata en la cámara de representantes, como resultado de las elecciones intermedias, impide que se dé por descontada su aprobación.

Por lo demás, las relaciones económicas continuaron su marcha ascendente a lo largo del sexenio en cuestión. Así, la inversión estadounidense en nuestro país ascendió a 71454 millones de dólares, y la mexicana en Estados Unidos a 5260 (contra 61233 millones de dólares, y 7190 millones de dólares del sexenio anterior, respectivamente). ${ }^{48}$ De manera similar, el intercambio comercial entre México y Estados Unidos mantuvo un crecimiento constante durante el gobierno peñanietista, a excepción de 2015 y 2016. En el sexenio (hasta 2017), México mantuvo un superávit comercial promedio con Estados Unidos de 122543 millones de dólares (contra 86452.7 millones de dólares del sexenio anterior). ${ }^{49}$

\section{RELACión CON CANADÁ}

Como de costumbre, México y Canadá mantuvieron buenas -si bien no intensas- relaciones durante el sexenio del presidente Enrique Peña Nieto; quizás los momentos de mayor

${ }^{47}$ K. Smith, "Oportunidades económicas y comerciales para México", Conferencia en el Centro Universitario de Ciencias Económicas y Administrativas, 10 de octubre de 2018.

${ }^{48}$ Secretaría de Economía, Inversión Directa de Estados Unidos hacia México, Dirección General de Inversión Extranjera de Secretaría de Economía; Bureau of Economic Analysis, U.S. Department of Commerce, http://www.bea.gov

${ }^{49}$ Secretaría de Economía, http://www.economia.gob.mx 
intensidad tuvieron lugar durante el periodo de renegociación del TLCAN (agosto de 2016-septiembre de 2018). De manera general, la relación bilateral siguió la pauta marcada por los tres gobiernos anteriores, los cuales mantuvieron contactos cordiales con Ottawa, pero no propusieron iniciativas de gran calado. Así, la Alianza México-Canadá, establecida en 2004, continuó las labores de colaboración durante el gobierno peñanietista, concentrándose en áreas como agroindustria, capital humano, comercio, energía, medio ambiente, minería movilidad laboral y silvicultura. Entre los logros alcanzados en estos rubros están Proyecta 10,000, iniciativa mexicana para incrementar el número de estudiantes mexicanos en Canadá, así como el acuerdo de movilidad en investigación industrial, firmado en 2016 por la organización no gubernamental canadiense Mitacs y el Consejo Nacional de Ciencia y Tecnología (Conacyt), mediante el cual hasta veinte estudiantes de posgrado de Canadá y México podrían hacer una estancia académica en una universidad del otro país. ${ }^{50}$ También en 2016 México levantó la prohibición a la importación de carne de res canadiense, y estableció una ventanilla única para la resolución de asuntos legales enfrentados por la mineras canadienses en nuestro país, las cuales representan aproximadamente $70 \%$ de la inversión extranjera en el sector. ${ }^{51}$

En el gobierno que nos ocupa, también se actualizó el convenio sobre transporte aéreo que regulaba las relaciones en la materia desde 1961; la nueva normatividad elimina impedimentos referentes a la frecuencia, capacidad y cantidad de aerolíneas en las denominadas tercera y cuarta libertades.

${ }^{50}$ Government of Canada,"Canada-Mexico partnership-2016 Annual Report” (en línea), https://www.canadainternational.gc.ca/mexico-mexi que/2016cmp-pcm.aspx?lang=eng (consulta de abril de 2017).

51 Government of Canada, "Address by Minister Dion at a news conference following the Canada-Mexico High-Level Strategic Dialogue meeting”, 12 de octubre de 2016, https://www.canada.ca/en/global-affairs/ news / 2016/10/address-minister-dion-news-conference-following-cana da-mexico-high-level-strategic-dialogue-meeting.html 
De esta manera, las compañías aéreas de Canadá y México pueden realizar operaciones entre cualquier par de ciudades del otro país. ${ }^{52}$ En el marco de la cooperación en materia de seguridad iniciada en el sexenio anterior, como una especie de efecto secundario de la Iniciativa Mérida, Ottawa continuó apoyando a nuestro país mediante el Programa de Desarrollo de Capacidades Contra el Crimen, particularmente en los que se refiere al proceso de reforma judicial y a la trata de personas. ${ }^{53}$ Resulta significativa la eliminación del requisito de visa a ciudadanos mexicanos que viajan a Canadá, situación impuesta en 2009 que, en su momento, fue motivo de fricción entre los gobiernos de Stephen Harper y Felipe Calderón. La nueva medida, que entró en vigor en diciembre de 2016, constituyó un importante mensaje de normalización de la relación entre dos países que son socios comerciales, pero además tuvo un efecto inmediato en el incremento de turistas mexicanos al vecino septentrional (tan sólo en la primera mitad de 2017, el número de viajeros casi había igualado el de 2016 en su totalidad). ${ }^{54}$

Pero como lo mencioné anteriormente, acaso los momentos más intensos de la relación entre "los vecinos del vecino" fueron aquellos que se vivieron en el contexto de la renegociación del tlcan. De entrada, Ciudad de México y Ottawa parecían estar en sincronía, con la intención de mostrar un frente unido ante Washington. Sin embargo, Canadá pronto pareció volver a su tradicional celo por mantener su "relación especial" con su vecino del sur. Ya durante los go-

52 Secretaría de Relaciones Exteriores, "Visita a México del Primer Ministro de Canadá, Stephen Harper", 18 de febrero de 2014; "México y Canadá firman modificaciones a acuerdo aéreo", El Economista, 2 de julio de 2017.

${ }^{53}$ Government of Canada, "Cooperación entre México y Canadá en el ámbito de la seguridad y la defensa”, 28 de junio de 2016, https:/ /www. canadainternational.gc.ca/mexico-mexique/media-presse/news-com muniques_160628-8.aspx?lang=es

${ }^{54}$ E. Peña Nieto, "Let's keep our Mexico-Canada relationship strong", The Globe and Mail, 12 de octubre de 2017. 
biernos de Calderón y Harper, importantes actores y líderes de opinión canadienses habían argumentado que el creciente acercamiento a México, en el marco del tratado comercial trilateral, podía "contaminar" la privilegiada relación que, en su opinión, su país mantenía con Washington. ${ }^{55}$ En esta ocasión no hubo referencia explícita a la cercanía entre Ottawa y Washington, sino que tanto la canciller y jefa negociadora, Chrystia Freeland, como el embajador canadiense en Washington, David MacNaughton, hicieron ver que su país estaba dispuesto a llegar a un entendimiento bilateral con Washington; como justificó Freeland, "nuestra relación con Estados Unidos es principalmente una relación bilateral". ${ }^{56}$

Sin embargo, durante la mayor parte del proceso, los equipos negociadores de Canadá y México mantuvieron una estrecha colaboración. ${ }^{57}$ De manera paradójica, conforme se acercaba la fecha límite para concluir las pláticas (el ejecutivo estadounidense tenía que notificar al congreso su intención de negociar el acuerdo comercial antes del $1^{\circ}$ de septiembre) fue México el que terminó negociando por separado con Estados Unidos; los dos países anunciaron su entendimiento el 27 de agosto. La noticia no cayó bien en algunos círculos canadienses $;{ }^{58}$ no obstante, su equipo continuó las negociaciones con Washington, y finalmente se anunció el nuevo TLCAN el 1 de octubre. El distanciamiento final en las negociaciones, sin embargo, no afectó la relación entre Ottawa y Ciudad de México; la canciller incluso reconoció

55 John Morrissy, "Canada Must Stress Trade Relationship with U.S. not NAFTA: Report”, The Financial Post, 11 de mayo de 2009; Andrés Rozental y Robert Pastor, "A Case of Three Amigos", The Globe and Mail, $1^{\circ}$ de mayo de 2009.

56 J. Díaz, "Considera Canadá acuerdo sin México", Reforma, 24 de enero de 2017.

${ }^{57}$ P. Viera, W. Mauldin, J. Montes, "Canada and Mexico Seek to Head Off U.S. Exit From NAFTA at Montreal Talks", The Wall Street Journal, 22 de enero de 2018.

${ }^{58}$ K. Yakabuski, "Chrystia Freeland's so-called friends in Mexico bid her adios on NAFTA", The Globe and Mail, 29 de agosto de 2018. 
a los negociadores mexicanos por haber facilitado el proceso entre su país y Estados Unidos, y México, por su parte, apoyó a Canadá en noviembre, durante las negociaciones del nuevo Acuerdo Transpacífico -sin Estados Unidos- llamado ahora Tratado Integral y Progresista de Asociación Transpacífico. 59

En el ámbito económico, la posición de Canadá como socio importante de nuestro país se consolidó durante el gobierno de Peña Nieto. Continuamos siendo el tercer socio comercial de Canadá. ${ }^{60}$ México mantuvo un superávit promedio de 848871 millones de dólares; cabe notar, sin embargo, que el comercio bilateral representa apenas $2.6 \%$ del comercio total de México y $3.4 \%$ del de Canadá. ${ }^{61}$ Por otra parte, Ottawa fue el cuarto inversionista en nuestro país (figura en los sectores que corresponden a la minería, transportes y correo, y servicios financieros). El promedio de inversión por año (2013-2017) aumentó $13.7 \%$ con respecto al sexenio anterior, sumando un total de 14185.9 millones de dólares. ${ }^{62}$

Un área de cooperación bilateral que se ha mantenido de manera exitosa es el Programa de Trabajadores Agrícolas Temporales, el cual data de 1974. Durante el periodo gubernamental que nos ocupa, el número de trabajadores mexicanos que participan en el programa se incrementó en 18000 ,

59 "Facilitó México pláticas EU-Canadá en TLC", Mural, 6 de septiembre de 2018; A. Blatchford, "How Canada allied with Mexico to fend off тTP pressure from Japan, Australia”, The Globe and Mail, 11 de noviembre de 2017.

60 "International merchandise trade for all countries and by Principal Trading Partners, monthly (x 1,000,000)", Statistics Canada (base de datos en línea), https://www150.statcan.gc.ca/t1/tbl1/en/tv.action?pid=1210 001101

${ }^{61}$ Statistics Canada, Balance del comercio canadiense en relación con México (base de datos en línea), https://www.ic.gc.ca/

${ }^{62}$ Secretaría de Economía, "Inversión Directa de Canadá hacia México", México, Dirección General de Inversión Extranjera, Secretaría de Economía, 2018. 
llegando a un total de 109067, es decir, un aumento aproximado de $20 \%$ respecto al sexenio anterior. ${ }^{63}$

\section{Política hacia América del Norte Y CONSIDERACIONES FINALES}

América del Norte, como región, no ocupó un lugar prominente en la política exterior del presidente Enrique Peña Nieto. En el Plan Nacional de Desarrollo, la región encabeza tan sólo una estrategia, la 5.1.1, cuyo objetivo es "Consolidar la relación con Estados Unidos y Canadá a partir de una visión integral y de largo plazo que promueva la competitividad y la convergencia en la región, sobre la base de complementariedades existentes", dentro del apartado "México con Responsabilidad Global". El modesto objetivo de consolidar la relación con los vecinos se estaba cumpliendo de forma razonable hasta la llegada de Donald Trump a la Casa Blanca en enero de 2017.

Así, en la Cumbre de Líderes de América del Norte, los mandatarios de los tres países se reunieron en febrero de 2014 en Toluca (Harper, Obama y Peña Nieto), y en junio de 2016 en Ottawa (Obama, Peña Nieto y Trudeau). En ambos encuentros, el énfasis se puso en temas como cambio climático, competitividad regional, defensa, energía limpia, seguridad. La actualización de la integración económica por medio del fallido acuerdo transpacífico era también un tema de la agenda compartida; como el presidente Peña Nieto declaró durante su visita de Estado a Canadá, en la víspera de la cumbre trilateral: el "TPP viene a revitalizar el acuerdo celebrado por NAFTA, a ponerle en otra escala y en otro nivel". ${ }^{64}$

63 Secretaría de Relaciones Exteriores, Informes de labores 20132018; Patricia Muñoz Ríos, “Trabajarán en Canadá 26 mil jornaleros agrícolas durante 2018”, La Jornada, 18 de enero de 2018.

${ }^{64}$ F. Reséndiz, F, "Sólida, integración de América del Norte: EPN”, El Universal, 27 de junio de 2016. 
Más allá de los planes de gobierno y de las reuniones de líderes, la relación con los otros dos países de América del Norte avanzó en buena medida a través de las redes transgubernamentales establecidas en los pocos temas en los que existe una agenda trilateral. De manera significativa, destaca de nueva cuenta el ámbito de la seguridad, en el cual los tres países dieron seguimiento a lo ya avanzado en años anteriores, por ejemplo de dos reuniones trilaterales de los ministros de defensa de América del Norte: la primera celebrada en abril de 2014 en la Ciudad de México y, la segunda, en mayo de 2017 (ya con Trump en la Casa Blanca) en Washington (el ejercicio se había inaugurado en marzo de 2012, cuando se celebró la primera reunión de este tipo en Ottawa).

El gobierno del presidente Peña trabajó también con sus socios en la coordinación diplomática de foros y temas extrarregionales. Así, desde 2014 (y hasta 2016) se llevaron a cabo reuniones del Mecanismo de Consultas Trilaterales sobre Temas Multilaterales; la temática incluyó áreas como derechos humanos, la ONU, refugiados y salud pública. ${ }^{65}$ Canadá-Estados Unidos y México también participaron, como región, en el Diálogo de América del Norte-Sistema de Integración Latinoamericana sobre seguridad. Otro impulso a la integración trilateral vino como efecto de las "reformas estructurales" aprobadas durante el gobierno que nos ocupa, particularmente la referente al sector energético. A raíz de esta última no sólo se han incrementado sustancialmente las inversiones en esa área provenientes de los otros dos países de la región, sino que el T-MEc cuenta con un capítulo en la materia, algo de lo que el tratado original carecía. ${ }^{66}$

Así pues, aunque el gobierno de Enrique Peña Nieto no tuvo una agenda ambiciosa (la cual, por otra parte, de haber existido no tenía garantizada su consecución, como sucedió

${ }^{65}$ Embajada de México en Estados Unidos, "México, Canadá y Estados Unidos celebran consultas trilaterales”, 3 de junio de 2015.

${ }^{66}$ R. Morales, "IED energética avanza sólida en enero-marzo", El Economista, 7 de junio de 2016; J. Miranda, "Transnacionales van por el sector energético”, La Jornada, 15 de septiembre de 2018. 
durante el gobierno de Vicente Fox $)^{67}$ pudo avanzar en la deseada consolidación de la relación con los dos socios de América del Norte -al menos, como apunté anteriormente, hasta antes de la llegada de Trump a la presidencia de Estados Unidos-. Si bien desde 2017 el gobierno mexicano se concentró en defender la frontera de política pública establecida con el TLCAN, a lo largo de todo el sexenio se normalizó -fundamentalmente a través de redes transgubernamentales- otra frontera de política pública, establecida recientemente durante el gobierno anterior: la cooperación en materia de seguridad con Estados Unidos. Más allá de las iniciativas llevadas a cabo por el gobierno de Enrique Peña Nieto respecto a las fronteras de política pública mencionadas, es de esperarse que una vez que pase (porque difícilmente llegó para quedarse) el torbellino que para la región ha representado el gobierno de Donald Trump, América del Norte podrá seguir avanzando en esquemas de cooperación e integración.

\section{Bibliografía}

Allen, B., "Trump's Trade Proposals Are so Obviously Unpopular They Give Mexicans Hope", Foreign Policy, 26 de octubre de 2017.

BAptista, D., “«Amarran» fondos de Iniciativa Mérida”, Mural, 29 de enero de 2017.

Berthame, Lee, "Trudeau, Mexican president stand up for free trade, take shots at Trump-style protectionism", The National Post, 28 de junio de 2016.

Cervantes, E., "Tuvo Trump actitud abierta y tolerante-Amlo", $R \boldsymbol{e}$ forma, $1^{\circ}$ octubre de 2018.

Clark, P., "Is the Trans Pacific Partnership a re-writing of Nafta? Is the Trans Pacific Partnership a re-writing of NAFTA?", iPolitics, 10 de enero de 2012.

67 Arturo Santa-Cruz, Mexico-United States Relations: The Semantics of Sovereignty, Nueva York: Routledge, 2012. 
Deare, C., A Tale of Two Eagles: The US-Mexico Bilateral Defense Relationship Post Cold War, Lanham, Rowman \& Littefield, 2017.

Díaz, J., "Considera Canadá acuerdo sin México", Reforma, 24 de enero de 2017.

Estévez, Dolia, "Cumbre militar en Cozumel, escenario del debut de México como líder regional”, Sin Embargo, 21 abril de 2017.

Faus, J., "El jefe del Pentágono viaja a México para celebrar el Día de la Independencia", El País, 15 de septiembre de 2017.

Golob, S., "Beyond the Policy Frontier: Canada, Mexico, and the Ideological Origins of NAFTA”. World Politics 55 (2003), pp. 361-398.

Guevara, M., A Bond Worth Strengthening: Understanding the Mexican Military and U.S.-Mexican Military Cooperation, Wilson Center Mexico Institute, 2016.

Guevara, I., "Análisis: La relación militar entre México-Eu, un oasis en la era Trump", El Heraldo de México, $1^{\circ}$ de mayo de 2017.

Hernández, É., "Destaca Presidente Integración con EeuU", $M u$ ral, 5 de febrero de 2016.

Hale, G., "Transnationalism, Transgovernmentalism and CanadaUS Relations in the Twenty-first Century", American Review of Canadian Studies, núm. 43 (2013), pp. 494-451.

Hope, A. Plus Ca Change: Structural Continuities in Mexican Counternarcotics Policy, Washington, DC, Brookings Institution, Latin American Initiative, 2016, https://www.brookings.edu/wpcontent/uploads/2016/07/Hope-Mexico-final.pdf

Hope, A., "Seis apuntes sobre un sexenio fallido", El Universal, 26 de noviembre de 2018.

Ivison, J., "White House advisors called Ottawa to urge Trudeau to help talk Trump down from scrapping NAFTA", National Post, 8 de mayo de 2018.

Keck, M. y K. Sikkink, Activists Beyond Borders: Advocacy Networks in International Politics, Ithaca, Cornell University Press, 1998.

Keck, M. y K. Sikкınk, "Transnational Advocacy Networks in the Movement Society”, en D. Meyer y S. Tarrow (eds.), The Social Movement Society: Contentious Politics for a New Century, Lanham, Rowman \& Littlefield, 1998, pp. 217-238. 
Keohane, R. y J. Nye, Transnational Relations and World Politics. Cambridge, Harvard University Press, 1972.

Keohane, R. y J. Nye, Power and Interdependence: World Politics in Transition. Boston, Little, Brown and Co., 1977.

López, L., "Iniciativa Mérida entregó 2.3 mil mdd en siete años", Milenio, 2 de febrero de 2017.

Lynch, D. y H. Long, "Analysts say Trump may be overly optimistic on new North American trade deal”, The Washington Post, $1^{\circ}$ de octubre de 2018.

Mintz, A. y S. REDD, "Framing Effects in International Relations". Synthese 135(2003), pp. 193-213.

Miranda, J., "Transnacionales van por el sector energético", $L a$ Jornada, 15 de septiembre de 2018.

Morales, R., "IED energética avanza sólida en enero-marzo, El Economista, 7 de junio de 2016.

Morrissy, J., "Canada Must Stress Trade Relationship with us not nAFTA: Report”, The Financial Post, 11 de mayo de 2009.

Mosso, R., "PGR y DEA, contra las redes financieras del narco en EU", Milenio, 16 de agosto 2018.

Ochoa,C. y E. Gut, “T-Mec es $20 \%$ mejor que el tlcan: Seade”, Milenio, 22 de octubre de 2018.

Muñoz Ríos, Patricia, “Trabajarán en Canadá 26 mil jornaleros agrícolas durante 2018”, La Jornada, 18 de enero de 2018.

Partlow, J. y S. Harowitz, "Us and Mexican authorities detail coordinated effort to capture drug lord", The Washington Post, 23 de febrero de 2014.

Partlow J. y N. Miroff, "U.s. gathers data on migrants deep in Mexico, a sensitive program Trump's rhetoric could put at risk", The Washington Post, 6 de abril de 2018.

Peña Nieto, E., "Let's keep our Mexico-Canada relationship strong”, The Globe and Mail, 12 de octubre de 2017.

Randal, C Archibold y G. Thompson, "Capture of Mexican Crime Boss Appears to End a Brutal Chapter", The New York Times, 16 de julio de 2013.

Randall, Archibold y E. Malkin, "Mexico Shifts Focus From Drug War to Economy", The New York Times, 27 de noviembre de 2012. 
Rapoza, K., "NAFta Will Live another Day, And Be Based On Obama's тPр”, Forbes, 14 de abril de 2017.

Reséndiz F., "Sólida, integración de América del Norte: EPN", $E l$ Universal, 27 de junio de 2016.

Ritchie, G., "NAFTA gets a new name, but little else has changed", The Globe and Mail, 1 de octubre de 2018.

Riva Palacio, R., "La captura de la abuela", El Financiero, 3 de marzo de 2017.

Romero, V., B. Magaloni y A. Díaz-Cayeros, "The Mexican War on Drugs: Crime and the Limits of Government Persuasion", International Journal of Public Opinion Research, núm. 27 (2014), pp. 125-137.

Rozental A. y R. Pastor, "A Case of Three Amigos", The Globe and Mail, $1^{\circ}$ de mayo de 2009.

Santa-Cruz A., Mexico-United States Relations: The Semantics of Sovereignty, Nueva York, Routledge, 2012.

Santa Cruz, A. "La política exterior de Felipe Calderón hacia América del Norte: crisis interna y redefinición de fronteras", Foro Internacional, vol. 53, núm. 213-214 (2013), pp. 537-571.

Schlesinger, J. y W. Mauldin , "Nafta Partners Prolong Talks Into Next Year", The Wall Street Journal, 17 de octubre de 2017. Secretaría de Relaciones Exteriores, "Visita a México del Primer Ministro de Canadá, Stephen Harper”, mex-can.sre.gob.mx "México y Canadá firman modificaciones a acuerdo aéreo", El Economista, 2 de julio de 2017.

Secretaría de Economía. Inversión Directa de Estados Unidos hacia México, México, Dirección General de Inversión Extranjera, Secretaría de Economía, 2018.

Seelke, C. y K. Finklea, U.s. Mexican Security Cooperation: The Merida Initiative and Beyond, Congressional Research Service, Report 7-5700, 2017.

Selee, A., Vanishing Frontiers: The Forces Driving Mexico and the United States Together, Nueva York, Public Affairs, 2018.

Shear, M. y R. Archibold, "Obama Arrives in Mexican Capital to Meet With New Leader”, The New York Times, 2 de mayo de 2013. Slaughter, A., "The Real New World Order", Foreign Affairs 76 (1997), pp. 183-197. 
Tolentino, J., "Seade: T-Mec no fue el mejor tratado, pero evitó una crisis financiera”, Expansión, 23 de octubre de 2018.

Tankersley, J. “Trump Just Ripped Up nafta. Here's What's in the New Deal”, The New York Times, $1^{\circ}$ de octubre 2018.

Vega, G. y F. Campos , La integración económica en América del Norte ante la elección de Donald Trump: un análisis prospectivo sobre el futuro del TLCAN, Foro Internacional, vol. 57, núm. 23057 (2017), pp. 781-836.

Viera, P., W. Mauldin y J. Montes, "Canada and Mexico Seek to Head Off U.s. Exit From nAfTA at Montreal Talks", The Wall Street Journal, 22 de enero de 2018.

Wilkinson, T., "Mexico captures drug kingpin's father-in-law", Los Angeles Times, 30 de abril de 2013.

Yakabuski, K., "Chrystia Freeland's so-called friends in Mexico bud her adios on NAFTA", The Globe and Mail, 29 agosto de 2018.

Informes, leyes e instrumentos internacionales

Secretaría de Relaciones Exteriores, Informe de Labores, 2012-2013, México, SRE.

Secretaría de Relaciones Exteriores, Informe de Labores, 2013-2014, México, SRE.

Secretaría de Relaciones Exteriores, Informe de Labores, 2014-2015, México, SRE.

Secretaría de Relaciones Exteriores, Informe de Labores, 2015-2016, México, SRE.

Secretaría de Relaciones Exteriores, Informe de Labores, 2016-2017, México, SRE.

Secretaría de Relaciones Exteriores, Informe de Labores, 2017-2018, México, SRE. 
